\title{
JUICIO MORAL Y ACTITUD AMBIENTAL DE LOS ALUMNOS DE QUINTO GRADO DE EDUCACIÓN SECUNDARIA DE BARRANCO
}

\author{
MORAL JUDGMENT AND ENVIRONMENTAL ATTITUDE OF FIFTH GRADE \\ HIGH SCHOOL STUDENTS FROM BARRANCO
}

\author{
Aldo Chumbe R. ${ }^{1}$ \\ Universidad Nacional Mayor de San Marcos, Lima, Perú \\ (RECibido el 02/11/2011, ACEPTADo El 22/12/ 2011)
}

\begin{abstract}
RESUMEN
El objetivo principal de esta investigación es determinar si existe una relación significativa entre el juicio moral y la actitud ambiental, según el tipo de gestión educativa, el género y la edad. Se emplea el diseño correlacional. La muestra representativa está conformada por 238 alumnos de quinto grado de educación secundaria del distrito de Barranco de Lima. Se aplican los instrumentos: Cuestionario de Reflexión Socio Moral (SROM) y Escala de Actitud Ambiental (ESACAMB). El análisis de datos se desarrolla mediante el programa SPSS 15.0 y con los estadísticos de la r de Pearson, chi-cuadrado, t de Student y ANOVA. Se concluye que existe una correlación significativa entre el juicio moral y la actitud ambiental. Respecto al juicio moral, los alumnos se ubican en la tercera etapa de la teoría de Kohlberg; los alumnos de centros educativos no estatales evidencian mayor juicio moral que los alumnos de estatales; no se hallan diferencias significativas según el género ni la edad. En relación a la actitud ambiental, los estudiantes se ubican en un nivel alto; las mujeres superan significativamente a los varones; y no se encuentran diferencias significativas de acuerdo al tipo de gestión educativa ni a la edad.
\end{abstract}

Palabras clave: Juicio moral, desarrollo moral, actitud ambiental, adolescencia, centro educativo estatal, centro educativo no estatal, género, edad.

\begin{abstract}
The main objective of this research is to determine whether there is a significant relationship between moral judgments and environmental attitude, according to the type of educational management, gender and age. Correlational design is used. The representative sample consists of 238 fifth grade students of secondary education Barranco district of Lima. Instruments are applied: Socio Moral Reflection Questionnaire (SROM) and Environmental Attitude Scale (ESACAMB). Data analysis is developed using SPSS 15.0 statistical and the Pearson's r, chi-square, Student $t$ test and ANOVA. We conclude that there is significant correlation

1 Magister en Psicología Educativa, egresado de la UPG de la Universidad Nacional Mayor de San Marcos E-maill: E-mail: aldo22deapril@hotmail.com
\end{abstract}


between moral judgments and environmental attitude. With regard to moral judgments, students are placed in the third stage of Kohlberg's theory, the students of non-state schools demonstrate greater moral judgments state students, no significant differences by gender or age. Regarding the environmental attitude, students are placed at a high level, with women significantly outperform men, and there are no significant differences according to type of educational management or age.

Keywords: moral judgments, moral development, environmental attitude, adolescence, state colleges, non-state school, gender, age.

\section{INTRODUCCIÓN}

La moralidad ha sido estudiada desde tiempos remotos, sin embargo, desde hace varias décadas destacaron teorías como la psicoanalítica y la del aprendizaje social, las cuales se basaron primordialmente en lo afectivo y lo conductual, respectivamente. Por otro lado, los estudiosos de la teoría cognoscitiva del desarrollo moral señalan que el crecimiento de la moralidad se da paralelamente al desarrollo del pensamiento, además de las interacciones del individuo con su entorno; por ello, el presente trabajo tomó como base la teoría del desarrollo moral de Kohlberg, cuya mayor contribución fue adoptar el concepto de desarrollo en etapas que Piaget construyó para el desarrollo cognitivo, por lo tanto, según Hers, Paolitto y Reimer, 1984, el juicio moral es un proceso cognitivo que nos permite reflexionar sobre nuestros valores y ordenarlos en una jerarquía lógica. Asimismo, Kohlberg plantea la existencia de tres niveles de juicio moral, las cuales constan de dos etapas cada una. El primer nivel, preconvencional, se caracteriza por los intereses concretos en base a consecuencias inmediatas; el segundo nivel, convencional, se basa en las expectativas de los demás o del mantenimiento del sistema social; el tercer nivel, posconvencional, se destaca por la autonomía moral basada en principios universales. En la etapa 1, principalmente el individuo actúa para evitar un castigo o recibir un premio; en la etapa 2, el individuo considera que tanto él como las demás personas pueden actuar para obtener un beneficio inmediato; en la etapa 3, la persona busca la aceptación social, es decir, ser reconocido como un buen hijo, buen amigo, etc.; en la etapa 4, la persona reconoce que deben mantenerse las normas sociales a como de lugar, porque de lo contrario se afectaría el equilibrio de la sociedad; en la etapa 5, se identifica que ante un conflicto entre las normas legales y morales, éstas deben anteponerse; mientras que en la etapa 6, la persona se rige por los principios universales, como: derecho a la vida, la paz, la igualdad, etc. Múltiples estudios sobre el juicio moral en adolescentes señalan que no existen diferencias significativas según el género (masculino y femenino). No obstante, algunos estudios muestran lo contrario, como por ejemplo: Rojas (1995); Velásquez (2007), referido por Giraldo, 2010; etc., en estas investigaciones usualmente las mujeres obtienen mayores puntajes. En relación al tipo de gestión educativa (centros educativos estatales y no estales), la mayoría de trabajos indica que los estudiantes de colegios no estatales presentan un mayor juicio moral. Asimismo, se destaca claramente que a mayor edad existe un mayor nivel de juicio moral. Respecto a la actitud ambiental, se 
adoptó la definición tridimensional de la actitud como "predisposición a responder a alguna clase de estímulo con alguna clase de respuesta". Estas respuestas pueden ser afectivas (sentimiento de agrado o desagrado), cognitivas (creencias $\mathrm{u}$ opiniones) y conductuales (intenciones conductuales o acciones manifiestas). De esta manera, la actitud se considera un concepto global formado por tres componentes relacionados entre sí: cognitivo, afectivo y conductual. Es decir, la actitud hacia la conservación del medio ambiente estaría compuesta por las creencias o cogniciones que mantenemos al respecto, el afecto que sentimos hacia el entorno natural y el comportamiento ecológico (Cialdini, Petty y Cacioppo, 1981, referidos por Aragonés y Amérigo, 2002). Además, la actitud ambiental se considera como la tendencia a responder favorablemente ante la conservación del medio o ante acciones y compromisos conductuales que favorezcan la conservación (Corral-Verdugo, 2001). Conforme a Corral-Verdugo (2001), es posible resumir en siete los principales problemas que enfrenta la biósfera terrestre: la atmósfera, los suelos (incluidos bosques), el agua, la basura (residuos sólidos), la energía, la biodiversidad y la población. Es importante obtener mayor conocimiento sobre la actitud ambiental de dicha actitud en distintos lugares, tal como se expone en un estudio de Gifford et al. (2009): "Sin embargo, las actitudes del medio ambiente y la preocupación están lejos de ser uniformes en todos los países (Franzen, 2003; Schultz y Zelezny, 1999) y la mayor investigación es necesaria para entender las formas en que el medio ambiente y las actitudes difieren en todo el mundo". Tal es así que Yarlequé (2004), en el Perú, ya había realizado un estudio sobre actitudes hacia la conservación ambiental en estudiantes de educación secundaria, se evidenció diferencias por el lugar de residencia y género. Recientemente en el 2009, Rivera y Rodríguez desarrollaron un estudio para determinar las actitudes y los comportamientos relacionados con salud ambiental en estudiantes de enfermería de una universidad del norte del Perú, se concluye que los participantes tienen una actitud ambiental positiva. Conjuntamente, otros autores señalan que los administradores o gerentes de empresas en corporaciones multinacionales (cuyas decisiones pueden afectar a un grupo o comunidad completa) poseen niveles de razonamiento moral más bajos que los normales de la población (Trevino, 1992, referido por Corral-Verdugo, 2001). Posteriormente, Biaggio et al. (1998), referidos por Corral-Verdugo, manifiestan que el nivel (elevado) de ese tipo de razonamiento pareciera ser una característica requerida para poseer actitudes proambientales. Además, Corral-Verdugo (2001) al realizar las conclusiones de unos estudios de personalidad ambiental, destaca que los individuos comprometidos con la conservación ambiental poseen un alto juicio moral. Nuévalos (2008), en España, llevó acabo un estudio correlacional donde halló una correlación entre el (juicio) criterio moral y los valores ambientales.

Actualmente se presentan problemas vinculados a la moralidad y al medio ambiente. De manera particular, en el distrito de Barranco existen colegios estatales y no estatales, los cuales cuentan con una gran población escolar adolescente de ambos géneros (masculino y femenino), quienes se encuentran expuestos a esos problemas, porque Barranco presenta dificultades como recuperación de los espacios públicos, 
tráfico vehicular en las principales avenidas, limpieza pública, mejora del ornato y seguridad ciudadana. Ante toda esta situación se propone como objetivo general:

Determinar si existe una relación significativa entre el juicio moral y la actitud ambiental de los alumnos de quinto grado de educación secundaria del distrito de Barranco, según el tipo de gestión educativa, el género y la edad.

Y como objetivos específicos:

1) Determinar en qué etapa del juicio moral se encuentran los alumnos de quinto grado de educación secundaria del distrito de Barranco.

2) Determinar si existen diferencias significativas en el juicio moral de los alumnos de quinto grado de educación secundaria del distrito de Barranco, según el tipo de gestión educativa, el género y la edad.

3) Determinar en qué nivel de la actitud ambiental se encuentran los alumnos de quinto grado de educación secundaria del distrito de Barranco.

4) Determinar si existen diferencias significativas en la actitud ambiental de los alumnos de quinto grado de educación secundaria del distrito de Barranco, según el tipo de gestión educativa, el género y la edad.

\section{MÉTODO}

El diseño es descriptivo comparativo, porque se identifican las características del juicio moral y la actitud ambiental, además de comparar sus etapas y niveles, respectivamente, de acuerdo a las variables sociodemográficas. También es correlacional al analizar el vínculo existente entre las dos variables según el tipo de gestión educativa, el género y la edad.

\section{Participantes}

Se emplea el muestreo no probabilístico y por criterio, además se aproximan los porcentajes de la muestra al de la población. La muestra está conformada por 238 alumnos de quinto grado de educación secundaria, procedentes de 11 centros educativos estatales y no estatales de Barranco.

\section{Instrumentos de recolección de datos}

Instrumento del juicio moral: Cuestionario de Reflexión Sociomoral (SROM). Autores: Gibbs y Widaman (1984). Adaptación: Mirian Grimaldo (2002). Administración: Individual o colectivamente a alumnos desde 14 años de edad. Tiempo de aplicación: 50 minutos, Aprox. Se halló la validez y confiabilidad en su país de origen (USA) y en la adaptación de Grimaldo (2002). En nuestro estudio, como parte de la validez, el análisis de medias evidencia una tendencia, a mayor edad, mayor puntaje. Para la confiabilidad, con el método Test-Retest, se obtiene una correlación de Pearson, que es significativa, positiva y alta $(r=0.81 \mathrm{p}<0.05)$.

Instrumento de la actitud ambiental: ESACAMB. Autores: El equipo de investigación. Administración: Individual o colectivamente a alumnos de 15, 16 y 17 años de edad de quinto grado de educación secundaria. Aplicación: 15 minutos, Aprox. La ESACAMB 
es una escala de actitud de tipo Likert que mide los niveles de actitud ambiental (bajo/ medio/alto), se organiza en 35 ítems con cinco alternativas cada uno. Para la validez se realiza la construcción con criterios estándar y se emplea el análisis psicométrico a través de la correlación ítem total corregida. La confiabilidad por consistencia interna se lleva a cabo mediante el coeficiente Alfa de Cronbach de la prueba total. El valor obtenido es de 0.84, lo cual indica que la ESACAMB es confiable.

\section{RESULTADOS}

Ho El puntaje directo del juicio moral no se correlaciona significativamente con el puntaje directo de la actitud ambiental de los alumnos de quinto grado de educación secundaria del distrito de Barranco, según el tipo de gestión educativa, el género y la edad.

Tabla 1. Correlación general entre los puntajes del juicio moral y la actitud ambiental de los alumnos

\begin{tabular}{cc}
\hline Variable & Puntaje de la actitud ambiental \\
$\mathrm{r}$
\end{tabular}

Puntaje del juicio moral

$0.32 * *$

$\mathbf{N}=\mathbf{2 3 8} \quad * \mathbf{p}<\mathbf{0 . 0 5} * * \mathbf{p}<\mathbf{0 . 0 1}$

En la tabla 1, se identifica que los puntajes del juicio moral y la actitud ambiental de los alumnos presentan una correlación significativa $(\mathrm{p}<0.05)$. Motivo por el cual, se rechaza la hipótesis nula (Ho).

Tabla 2. Correlación entre los puntajes del juicio moral y la actitud ambiental de los alumnos según el tipo de gestión educativa

\begin{tabular}{ccc}
\hline & \multicolumn{2}{c}{ Puntaje de la actitud ambiental } \\
\cline { 2 - 3 } Variable & Estatal & No estatal \\
& $\mathrm{N}=154$ & $\mathrm{~N}=84$ \\
& $\mathrm{r}$ & $\mathrm{r}$ \\
\hline
\end{tabular}

$\begin{array}{lll}\text { Puntaje del juicio moral } & 0.27 * * & 0.36 * *\end{array}$

\section{* $\mathrm{p}<0.05$ ** $\mathrm{p}<0.01$}

En la tabla 2, se muestra que los puntajes del juicio moral y la actitud ambiental, tanto de los alumnos de centros educativos estatales como de los no estatales, presentan una correlación significativa $(\mathrm{p}<0.05)$. Motivo por el cual, se rechaza la hipótesis nula (Ho). 
Tabla 3. Correlación entre los puntajes del juicio moral y la actitud ambiental de los alumnos según el género

\begin{tabular}{|c|c|c|}
\hline \multirow[b]{2}{*}{ Variables } & \multicolumn{2}{|c|}{ Puntaje de la actitud ambiental } \\
\hline & $\begin{array}{c}\text { Masculino } \\
\mathrm{N}=89 \\
\mathrm{r}\end{array}$ & $\begin{array}{c}\text { Femenino } \\
\mathrm{N}=149 \\
\mathrm{r}\end{array}$ \\
\hline Puntaje del juicio moral & 0.10 & $0.39 * *$ \\
\hline
\end{tabular}

En la tabla 3, se muestra que considerando el género masculino no existe una correlación significativa $(p>0.05)$; mientras que para el género femenino se presenta una correlación significativa $(\mathrm{p}<0.05)$. Motivo por el cual, respecto al género masculino, se acepta la hipótesis nula (Ho). Mientras que, sobre el género femenino, se rechaza la hipótesis nula (Ho).

Tabla 4. Correlación entre los puntajes del juicio moral y la actitud ambiental de los alumnos según la edad

\begin{tabular}{cccc}
\hline & \multicolumn{3}{c}{ Puntaje de la actitud ambiental } \\
\cline { 2 - 4 } Variable & 15 años & 16 años & 17 años \\
& $\mathrm{N}=51$ & $\mathrm{~N}=158$ & $\mathrm{~N}=29$ \\
$\mathrm{r}$ & $\mathrm{r}$ & $\mathrm{r}$ \\
\hline $\begin{array}{c}\text { Puntaje del juicio } \\
\text { moral }\end{array}$ & $0.33 *$ & $0.34 * *$ & 0.23 \\
\hline
\end{tabular}

$* \mathbf{p}<0.05 * * \mathrm{p}<0.01$

En la tabla 4, el coeficiente de correlación para los puntajes del juicio moral y la actitud ambiental, muestra que considerando los 15 y 16 años de edad se presenta una correlación significativa $(\mathrm{p}<0.05)$. Por otro lado, en la edad de 17 años no existe una correlación significativa ( $\mathrm{p}>0.05)$. Por ello, en relación a las edades de 15 y 16 años, se rechaza la hipótesis nula (Ho). Opuestamente, respecto a la edad de 17 años, se acepta la hipótesis nula (Ho).

Ho La proporción de sujetos distribuidos en las etapas del juicio moral ubica significativamente en la etapa 4 del juicio moral a los alumnos de quinto grado de educación secundaria del distrito de Barranco.

Tabla 5. Análisis y determinación de la etapa del juicio moral

\begin{tabular}{cccc}
\hline Etapa del juicio moral & N observado & N esperado & Residual \\
\hline 2 & 2 & 79.3 & -77.3 \\
3 & 173 & 79.3 & 93.7 \\
4 & 63 & 79.3 & -16.3 \\
Total & 238 & & \\
Chi-cuadrado(a) & 189.336 & & \\
gl & 2 & & \\
Sig. Asintót. & .000 & & \\
\hline
\end{tabular}


En la tabla 5, se aprecia que existen diferencias estadísticas significativas, pues el estadístico chi-cuadrado alcanza un valor de 189.336, el cual es significativo $(\mathrm{p}<0.05)$. Notándose un predomino de la etapa 3 del juicio moral. Motivo por el cual, se rechaza la hipótesis nula (Ho).

Ho El puntaje promedio del juicio moral de los alumnos de centros educativos estatales no presenta una diferencia significativa con el de los no estatales de quinto grado de educación secundaria de Barranco.

Tabla 6. Análisis comparativo de los puntajes del juicio moral según el tipo de gestión educativa

\begin{tabular}{ccccccc}
\hline $\begin{array}{c}\text { Tipo de } \\
\text { gestión }\end{array}$ & $\mathrm{N}$ & Media & Desviación típ. & $\begin{array}{c}\mathrm{t} \\
\text { Student }\end{array}$ & gl & $\begin{array}{c}\text { Sig. } \\
\text { (bilateral) }\end{array}$ \\
\hline Estatal & 154 & 321.42 & 31.37 & -3.52 & 236 & .001 \\
$\begin{array}{c}\text { No estatal } \\
\text { Total }\end{array}$ & 84 & 337.42 & 37.14 & & & \\
\hline
\end{tabular}

La prueba t de Student, en la tabla 6, demuestra que la diferencia entre los puntajes del juicio moral de los alumnos de centros educativos estatales y de los no estatales, es significativa $(\mathrm{p}<0.05)$. El puntaje de los alumnos de centros educativos no estatales es mayor que el de los alumnos de centros educativos estatales. Por tal motivo, se rechaza la hipótesis nula (Ho).

Ho El puntaje promedio del juicio moral de los alumnos del género masculino no presenta una diferencia significativa con el del género femenino de quinto grado de educación secundaria de Barranco.

Tabla 7. Análisis comparativo de los puntajes del juicio moral según el género

\begin{tabular}{ccccccc}
\hline Género & $\mathrm{N}$ & Media & $\begin{array}{c}\text { Desviación } \\
\text { típ. }\end{array}$ & $\begin{array}{c}\mathrm{t} \\
\text { Student }\end{array}$ & $\mathrm{gl}$ & $\begin{array}{c}\text { Sig. } \\
\text { (bilateral) }\end{array}$ \\
\hline Masculino & 89 & 321.76 & 35.22 & -1.852 & 236 & .065 \\
Femenino & 149 & 330.23 & 33.47 & & & \\
Total & 238 & & & & & \\
\hline
\end{tabular}

En la tabla 7, de acuerdo a la t de Student, se identifica que no existe una diferencia significativa entre los puntajes de los alumnos del género masculino y del género femenino. ( $\mathrm{p}>0.05)$. Por ello, se acepta la hipótesis nula (Ho).

Ho Los puntajes promedio del juicio moral de los alumnos de 15, 16 y 17 años de edad no presentan una diferencia significativa con los de las distintas edades estudiadas de los alumnos de quinto grado de educación secundaria del distrito de Barranco. 
Tabla 8. Análisis comparativo de los puntajes del juicio moral según la edad

\begin{tabular}{cccc}
\hline Edad & $\mathrm{N}$ & Media & Desviación típica \\
\hline 15 & 51 & 320.96 & 33.03 \\
16 & 158 & 328.27 & 33.40 \\
17 & 29 & 331.23 & 40.82 \\
Total & 238 & 327.07 & 34.31 \\
\hline F = 1.119 & & \\
Sig. $=328$ & & \\
\hline
\end{tabular}

En la tabla 8, a través del ANOVA, se determina que no existen diferencias significativas entre los puntajes del juicio moral de los alumnos de las distintas edades estudiadas $(\mathrm{p}>0.05)$. Por ello, se acepta la hipótesis nula (Ho).

Ho La proporción de sujetos distribuidos en los niveles de actitud ambiental ubica significativamente en el nivel alto a los alumnos de quinto grado de educación secundaria del distrito de Barranco.

Tabla 9. Análisis y determinación del nivel de la actitud ambiental

\begin{tabular}{cccc}
\hline $\begin{array}{c}\text { Nivel de actitud } \\
\text { ambiental }\end{array}$ & N observado & N esperado & Residual \\
\hline Medio & 36 & 119.0 & -83.0 \\
Alto & 202 & 119.0 & 83.0 \\
Total & 238 & & \\
\hline Chi-cuadrado(a) & 115.782 & & \\
gl & 1 & & \\
Sig. Asintót. & .0001 & & \\
\hline
\end{tabular}

En la tabla 9, se aprecia que existen diferencias significativas, pues el estadístico chi-cuadrado alcanza un valor de $115.782(\mathrm{p}<0.05)$. Notándose un predomino del nivel alto de actitud ambiental. Se acepta la hipótesis nula (Ho).

Ho El puntaje promedio de la actitud ambiental de los alumnos de centros educativos estatales no presenta una diferencia significativa con el de los no estatales de quinto grado de educación secundaria de Barranco.

Tabla 10. Análisis comparativo de los puntajes de la actitud ambiental según el tipo de gestión educativa

\begin{tabular}{ccccccc}
\hline $\begin{array}{c}\text { Tipo de } \\
\text { gestión }\end{array}$ & $\mathrm{N}$ & Media & $\begin{array}{c}\text { Desviación } \\
\text { típ. }\end{array}$ & $\begin{array}{c}\mathrm{t} \\
\text { Student }\end{array}$ & gl & $\begin{array}{c}\text { Sig. } \\
\text { (bilateral) }\end{array}$ \\
\hline Estatal & 154 & 136.16 & 13.82 & -1.341 & 236 & .181 \\
$\begin{array}{c}\text { No estatal } \\
\text { Total }\end{array}$ & 84 & 138.64 & 13.28 & & & \\
\hline
\end{tabular}


En la tabla 10, la t de Student evidencia que no existen diferencias significativas entre los puntajes de los alumnos de centros educativos estatales y de los no estatales ( $p>0.05)$. Por ello, se acepta la hipótesis nula (Ho).

Ho El puntaje promedio de la actitud ambiental de los alumnos del género masculino no presenta una diferencia significativa con el del género femenino de quinto grado de educación secundaria de Barranco.

Tabla 11. Análisis comparativo de los puntajes de la actitud ambiental según el género

\begin{tabular}{ccccccc}
\hline Género & $\mathrm{N}$ & Media & $\begin{array}{c}\text { Desviación } \\
\text { típ. }\end{array}$ & $\begin{array}{c}\mathrm{t} \\
\text { student }\end{array}$ & gl & $\begin{array}{c}\text { Sig. } \\
\text { (bilateral) }\end{array}$ \\
\hline Masculino & 89 & 131.71 & 10.672 & -4.872 & 236 & .000 \\
Femenino & 149 & 140.22 & 14.269 & & & \\
Total & 238 & & & & & \\
\hline
\end{tabular}

Mediante la t de Student, en la tabla 11, se observa que existen diferencias significativas entre los puntajes de la actitud ambiental de los alumnos del género masculino y los del género femenino $(\mathrm{p}<0.05)$. También se resalta que el puntaje de la actitud ambiental de las mujeres es mayor que el puntaje de los varones. En tal sentido, se rechaza la hipótesis nula (Ho).

Ho Los puntajes promedio de la actitud ambiental de los alumnos de 15, 16 y 17 años de edad no presentan una diferencia significativa con los de las distintas edades estudiadas de los alumnos de quinto grado de educación secundaria del distrito de Barranco.

Tabla 12.Análisis comparativo de los puntajes de la actitud ambiental según la edad

\begin{tabular}{cccc}
\hline Edad & $\mathrm{N}$ & Media & Desviación típica \\
\hline 15 & 51 & 136.73 & 13.075 \\
16 & 158 & 137.82 & 14.075 \\
17 & 29 & 133.34 & 12.025 \\
Total & 238 & 137.04 & 13.656 \\
\hline $\mathrm{F}=1.334$ & & & \\
Sig. $=.265$ & & & \\
\hline
\end{tabular}

En la tabla 12, a través del ANOVA se determina que no existen diferencias significativas entre los puntajes de la actitud ambiental de los alumnos de las distintas edades estudiadas ( $\mathrm{p}>0.05)$. Por ello, se acepta la hipótesis nula (Ho). 


\section{DISCUSIÓN}

Los análisis estadísticos mostraron que existe una relación significativa (y positiva) entre los puntajes del juicio moral y la actitud ambiental de los alumnos de quinto grado de educación secundaria del distrito de Barranco (Tabla 1). Este primer resultado coincide con el resultado obtenido por Nuévalos (2008), quien señaló que sus resultados brindarían apoyo a la idea de que los sujetos más desarrollados en razonamiento moral son, a la vez, los que tienen mayores actitudes positivas de respeto y cuidado con su entorno físico. En nuestro estudio también se identifica una relación significativa entre el juicio moral y la actitud ambiental, tanto para alumnos de centros educativos estatales como para los de no estatales (Tabla 2). Al abordar la correlación de nuestras variables por género (Tabla 3), se observa la similitud en la tendencia de los puntajes de las alumnas (género femenino), no obstante, se evidencia una ausencia de vínculo entre el juicio moral y la actitud ambiental de los varones. De igual manera, al indagar sobre las relaciones por edad (Tabla 4), se evidencia una correlación entre los puntajes del juicio moral y la actitud ambiental de los alumnos de 15 y 16 años. Algo contario ocurre en los alumnos de 17 años, cuyos puntajes de las variables no se correlacionan. Es decir, según nuestros análisis de correlación por tipo de gestión, género y edad, existen mayoritariamente correlaciones significativas.

Este estudio evidencia que los alumnos de quinto grado de educación secundaria del distrito de Barranco se ubican (por sus puntajes) en la etapa 3 del juicio moral (Tabla 5). A la vez, este resultado confirma lo obtenido por la mayoría de investigaciones donde se señala que el gran número de adolescentes se ubica en esta etapa (3). Al comparar los puntajes del juicio moral por el tipo de gestión educativa, se evidencia que existe una diferencia significativa (Tabla 6), lo cual concuerda con varios estudios que también encontraron dichas diferencias, como: Valdez, 1985, referido por Chumbe, 2004; Grimaldo, 1999; Vargas, 2007. Además, se presenta un mayor juicio moral por parte de los alumnos de centros educativos no estatales. Respecto a la diferencia del juicio moral por el género, se observa que no hay diferencias significativas entre los puntajes de las mujeres y los varones (Tabla 7). Aquí se ratifica lo hallado en múltiples estudios, por ejemplo: Ubaqui (2008); Grimaldo (2009); Giraldo (2010). Al culminar las comparaciones de los puntajes del juicio moral, se aprecia que no existen diferencias significativas entre los alumnos de 15, 16 y 17 años de edad (Tabla 8). Este resultado es similar a los obtenidos por los siguientes investigadores: Uculmana (1999); Ubaqui (2008); Giraldo (2010); asimismo, la explicación estaría en que se compararon a alumnos con edades cercanas, estos alumnos se encontrarían en la misma edad psicológica, es decir, el hecho de que los alumnos se encuentren en el mismo grado (quinto de secundaria) y, a la vez, estén expuestos prácticamente a las mismas experiencias (el estudio de ciertos temas educativos, las normas establecidas por otros, los intereses de sus coetáneos, etc.) puede influir en que no se logren apreciar diferencias significativas en su juicio moral. Sin embargo, debe mencionarse (o reiterarse) que si bien no hay una diferencia significativa por edad, se evidencia una tendencia positiva en los puntajes, ello permite apoyar la teoría de Kohlberg, la edad es un 
componente vinculado a los modelos del juicio moral, aunque no es determinante.

Los resultados sobre la actitud ambiental muestran que estadísticamente los alumnos de quinto grado de educación secundaria del distrito de Barranco se ubican en un alto nivel de actitud ambiental (Tabla 9). Esta actitud ambiental positiva se aprecia también en la encuesta del Instituto de Opinión Pública (2009), donde el $89 \%$ de los ciudadanos de Lima Metropolitana opina que el problema de la conservación del Medio Ambiente es muy importante. Al comparar los puntajes de la actitud ambiental por el tipo de gestión educativa, se aprecia que no existen diferencias significativas entre los alumnos de centros educativos estatales y no estatales (Tabla 10). Este resultado puede tener explicación en el hecho de que en los últimos años se viene brindando una mayor difusión sobre el tema de los problemas ambientales, es decir, la información proambientalista (y gradualmente los programas) está llegando a centros estatales como no estatales. Por ejemplo, el $60 \%$ de habitantes de Lima Metropolitana considera estar muy informado o por lo menos algo informado (Instituto de Opinión Pública, 2009); debe aclararse que, en efecto, la información no determina un comportamiento ambiental, pero sí es necesaria para lograr competencias proambientales. Otra de las causas de la inexistencia de diferencias en la actitud ambiental por el tipo de gestión educativa, sería la actual "presión social" hacia la conservación del medio ambiente, puesto que en la escuela, los medios de comunicación y otros ámbitos, son muy criticables aquellas acciones que pudiesen conllevar al deterioro del espacio que se habita o el mal uso de los recursos. La comparación de los puntajes de la actitud ambiental entre los varones y las mujeres, confirman nuestra hipótesis de que existen diferencias significativas según el género (Tabla 11); además, se aprecia que las mujeres tienen una mayor actitud ambiental que los varones. Se hallan múltiples investigaciones, al igual que fundamentos teóricos que permitirían avalar este resultado. Los investigadores Rivera y Rodríguez (2009), tal como los referidos por Corral-Verdugo (2001), entre otros, reportan que las mujeres muestran mayores niveles de actitud, preocupación o responsabilidad ambientales. Igualmente se presentan posturas e ideas importantes, como las del movimiento ecofeminista que plantea una afinidad singular entre la ecología y el feminismo, tal es así que las mujeres y la naturaleza comparten dos "experiencias" en común: 1) ambas tienen la capacidad de reproducir (dicha capacidad no pueden adquirirla los hombres) y 2) las dos se ubican en una posición subordinada dentro de la gran jerarquía patriarcal: Dios-hombre-mujer-naturaleza (Eckersley, 1992, referido por Corral-Verdugo, 2001). Al estudiar roles sexuales se plantea que las niñas adoptan tempranamente una percepción relacional y comunitaria del mundo, mientras que los niños se interesan por objetivizar y controlar su ambiente, autodefiniéndose como entidades apartadas de la naturaleza (Horrigan, 1989; Keller, 1985, referido por Corral-Verdugo). Los alumnos de 15, 16 y 17 años de edad de quinto grado de educación secundaria no muestran diferencias significativas entre sí, respecto a su actitud ambiental (Tabla 12). Este resultado se asemeja con lo encontrado por Yarlequé (2004), quien indicó que la edad fue poco relevante en la actitud ambiental de su trabajo de investigación. Por otro lado, nuevamente, se advierte 
que de acuerdo a la edad los alumnos no presentarían diferencias significativas, debido a que compartirían la misma edad psicológica al encontrase en el mismo grado (5to de secundaria) y estar expuestos a similares experiencias propias de sus coetáneos. Igualmente, otro de los factores sería la presión social.

\section{CONCLUSIONES}

1. Existe una correlación significativa entre el juicio moral y la actitud ambiental de los alumnos de quinto grado de educación secundaria de Barranco.

2. Se halla una correlación significativa entre las variables de estudio según el tipo de gestión educativa, es decir, para estatales y no estatales.

3. Se encuentra una correlación significativa entre las variables de estudio según el género femenino, mas no en el género masculino.

4. Entre las variables de estudio se evidencia una correlación significativa según las edades de 15 y 16 años. Pero no en la edad de 17 años.

5. Los alumnos de quinto grado de educación secundaria de Barranco se ubican en la tercera etapa y en el nivel convencional del juicio moral.

6. Se hallan diferencias significativas en el juicio moral según el tipo de gestión educativa a favor de los alumnos de centros educativos no estatales.

7. No se hallan diferencias significativas en el juicio moral según el género ni la edad.

8. Los alumnos de quinto grado de educación secundaria del distrito de Barranco se encuentran ubicados en un nivel alto de actitud ambiental.

9. Se hallan diferencias significativas según el género, las mujeres evidencian una superior actitud ambiental.

10. No se evidencian diferencias significativas en la actitud ambiental según el tipo de gestión educativa ni a la edad.

\section{REFERENCIAS BIBLIOGRÁFICAS}

1. Aragonés, J. y Amérigo, M. (2002). Psicología ambiental. Madrid: Pirámide.

2. Chumbe, A. (2004). Juicio moral en escolares de sexto grado de educación primaria de Lima Metropolitana. Tesis de titulación. Universidad Nacional de Educación “Enrique Guzmán y Valle”. Lima.

3. Chumbe, A. (2011). Juicio moral y actitud ambiental de los alumnos de quinto grado de educación secundaria de Barranco. Tesis de maestría. Universidad Nacional Mayor de San Marcos. Lima.

4. Corral-Verdugo, V. (2001). Comportamiento proambiental. Santa Cruz de TenerifeEspaña: Resma.

5. Gifford, R., Scannell, L., Kormos, C., Smolova, L., Biel, A., Boncu S., et al. (2009).Temporal pessimism and spatial optimism in environmental assessments: An 18-nation study. Journal of Environmental Psychology, vol.29, 1-12.

6. Giraldo. P. (2010). La autoestima y el juicio moral en los alumnos de secundaria de 
las instituciones educativas públicas de San Juan de Lurigancho. Tesis de maestría. Universidad Nacional Mayor de San Marcos. Lima.

7. Grimaldo, M. (1999). ¿Hacia dónde van nuestros jóvenes? Lima: Universidad de San Martín De Porres.

8. Grimaldo, M. (2009). Juicio moral en universitarios de la ciudad de Lima. [Versión electrónica], Psicogente, 12 (21): pp. 12-28. Recuperado el 9 de enero del 2011, de http://www.unisimonbolivar.edu.co/rdigital/ psicogente/index.php/psicogente/ article/viewFile/148/155

9. Hers, R., Paolitto, P. y Reimer, J. (1984). El crecimiento moral: De Piaget a Kohlberg. Madrid: Narcea.

10. Instituto de Opinión Pública (2009). Estado de la opinión pública: Medio ambiente. $P U C P$, año IV, junio, pp. 1-13.

11. Nuévalos, C. (2008). Desarrollo moral y valores ambientales. Tesis doctoral. Universidad de Valencia.

12. Rivera, J. y Rodríguez, C. (2009). Actitudes y comportamientos en estudiantes de enfermería de una universidad pública del norte, Revista Peruana de Medicina Experimental y Salud Pública, 26(3), pp.338-342. Recuperado el 13 de marzo del 2011, de http://www.scielo.org.pe/pdf/rins/v26n3/a12v26n3.pdf

13. Rojas, J. (1995). Juicio moral en una muestra de adolescentes del quinto año de secundaria pertenecientes a la USE 014 de Lima Metropolitana. Tesis de maestría. Universidad Nacional Mayor de San Marcos. Lima.

14. Ubaqui, P. (2008). Valores interpersonales y criterio moral en alumnos de quinto de secundaria de instituciones educativas de Chanchamayo. Tesis de maestría. Universidad Nacional de Centro del Perú. Huancayo.

15. Uculmana, C. (1999). Desarrollo moral, según edad, sexo e institución de procedencia. Tesis de maestría. Universidad de San Martín de Porres. Lima.

16. Vargas, J. (2007). Juicio moral y autoestima en estudiantes escolares del quinto año de secundaria de colegios de Lima Metropolitana. Tesis de maestría. Universidad Nacional Mayor de San Marcos. Lima.

17. Yarlequé, L. (2004). Actitudes hacia la conservación ambiental en estudiantes de educación secundaria. Tesis doctoral. Universidad Nacional Mayor de San Marcos. Lima. 